\title{
Herbal Medicine, Diabetes Care at Primary Care Facilities and Prevention of Vertical HIV Transmission
} Adamson S. Muula

Editor-in-Chief, Malawi Medical Journal, Professor and Head, Department of Public Health, The Kamuzu University of Health Sciences (KUHeS), Blantyre, Malawi

Email address: amuula@kuhes.ac.mw

In this September 2021 issue of the Malawi Medical Journal, three articles which attracted my attention are those by Mbali et al, ${ }^{1}$ Zimba et al, ${ }^{2}$ and Pfaff et al. ${ }^{3}$ Perhaps as Editor-in-Chief of the Journal, I should endeavor to be impartial and "love" all the articles the same. This time however, I have selected these articles because they touch on important health issues in Malawi, with Mbali et al, reporting on the use of herbal medicines among individuals living with HIV and receiving life-saving antiretroviral therapy (ART). In a country where about $8 \%$ of the adult population is estimated to be living with HIV, this is an important article which raises important issues on the use of complementary, herbal, or alternative medicine use among a large population of our society; and with positive, sometime negative and perhaps neutral consequences.

The second article I have chosen, i.e., Zimba et al is no less important. With close to half of the population being 18 years, and a total fertility rate of 4.4 , the reproductive momentum and its attendant impact on vertical (mother to child) transmission of HIV cannot be over-emphasised. Thus the article on the prevention of mother to child transmission of HIV by Zimba et al, is timely (others say timeous) and call for rigorous reflection as to what needs to be improved if the successes so far registered in the prevention of vertical HIV transmission (and acquisition) must be consolidated. Malawi has been a pacesetter with respect to research and practice in prevention of mother to child transmission. Research done in the country informed the global adoption of Option B programmes and later Option $\mathrm{B}+$ for the prevention of vertical HIV infection.

Pfaff et al reports on the situation, challenges and opportunities of increasing the covering of diabetes mellitus case management at the primary care level. A previous study had estimated that about $6 \%$ of adult Malawi may be living with diabetes. ${ }^{4}$ The quality of diabetes care in rural settings of Malawi has previously been faulted ${ }^{5}$ and in need of "some glucose bolus" and later, sustained release. And, if what the pundits (or is epidemiologists) are saying is true, this percentage is likely to increase unless something drastic happens. In fact the pundits have not said that these projections are going to be lower than feared, although as they say in medicine, "Never say never".

It is often estimated that $80 \%$ of people in Africa use herbal medicine. Where such an estimate came from can be debated but for now, although widely quoted, let us call it a guess. The Demographic and Health Surveys (DHSs) are among the most rigorous and national and regular surveys that many African countries conduct. These surveys emphasise on maternal and child health. Yet, such large national studies, or similarly designed surveys would have been those from which national herbal medicine use estimates could be obtained. The estimation of herbal medicine use in Africa has been haphazard to say the least. Studies have been conducted in specific settings and often with small sample sizes. Estimating national prevalence of herbal medicine use from these not so large enough and let alone for African as a whole is being less than candid. In sum, we have two choices. One, is to dispense of with the often-quoted $80 \%$ prevalence story and admit that we don't know. Second, if we feel it is important to estimate the prevalence of herbal medicines use, then we carry out appropriate studies that will enable us to answer such questions. Thirdly, we can attempt to be modest but at least fair by specifically providing the temporal, geographical and group characteristics of our studies in herbal medicine use. Mbali et al's article on herbal medicine use among individual accessing the ART programme at the Queen Elizabeth Central Hospital (QECH) in Blantyre, Malawi is specific to a particular population group and at one point in time. Whether herbal medicine use in the patient source population is higher or lower than what Mbali reported may be verified. Are individuals on the ART programme, more or less likely to use herbal medicines than the general populations? I don't know. Mbali et al found that $17.5 \%$ of the patients in her group were using herbal medicines and those who reported urban residence were less likely to report herbal medicine use. Where these urban dwellers are missing out from some benefit or avoiding harm cannot be inferred from Mbali et al.

Coming to the second article, I selected for this editorial, Zimba et al's paper on Option $\mathrm{B}+$ and prevention of mother to child transmission of HIV found that "staffing shortages, transportation challenges, limited space and infrastructure, limited stocks of HIV testing kits, and large patient populations" were some of the barriers against the optimal delivery of services. Sadly, we already know these barriers which seem recalcitrant (I got this word from former Australian Prime Minister Paul Keating when he, referred to Mahatir Mahamad as such in 1993). Many people can outline what needs to be done to ameliorate these health system challenges but wouldn't be specific as to the how. How can staffing shortages be addressed? Train more? Hire more? Retain more? Fund more? Where is the money going to come from? How do we reduce wastages? Why? What? How? When? And Which?

The Colin Pfaff et al paper is not unlike that by Zimba et 
$a l$, at least some respects. The shortages and limitations of cascading diabetes management down to the primary care facilities should be known to many readers. Limited numbers of staff. Staff competence not up to the task. Diagnostic supplies and drug shortages. Healthcare workers, and doctors especially, are educated and trained to solve problems. Readers of Pfaff et al must be geared up to solve the problems as outlined in the article, else what was the purpose for the article? It is heartening that Malawi has, since 2010, taken the path to improve non-communicable diseases research and practice or services. Some of the leadership in this regard have been from the research front (e.g Msyamboza $e t a)^{7,8}$, policy and institutional building (Dr Beatrice Matanje who was the inaugural lead at the Ministry of Health's NCD Unit) to Jones Kaponda-Masiye and from 2021 Jonathan Chiwanda. The country seems ready to address the gaps in the management of diabetes, among other non-communicable diseases. Those of us at the Malawi Medical Journal are looking forward to receiving and sending for review manuscripts that document the positive changes that we expect.

\section{References}

1. Mbali H, Sithole JJK, Nyondo-Mipando AL. Prevalence and correlates of herbal medicine use among antiretroviral (ART) clients at Queen Elizabeth Central Hospital (QECH) Blantyre Malawi: a cross sectional study. Malawi Med J, 2021
2. Zimba C, Sherwood G, Mark B, et al. Supporting the implementation of guidelines to prevent mother-to-child transmission of HIV in Malawi. A multi-case study. Malawi Med J, 2021

3. Pfaff C, Malamula g, Kamowatimwa G, et al. Decentralising diabetes care from hospitals to primary health care centers. Malawi Med J, 2021

4. Msyamboza KP, Mvula CJ, Kathyola D. Prevalence and correlates of diabetes mellitus in Malawi: population-based national NCD STEPS survey. BMC Endocr Disord. 2014 May 12;14:41. doi: 10.1186/14726823-14-41.

5. Assayed AA, Muula AS, Nyirenda MJ. The quality of care of diabetic patients in rural Malawi: A case of Mangochi district.Malawi Med J. 2014 Dec;26(4):109-14.

6. Msyamboza KP, Mvula CJ, Kathyola D. Prevalence and correlates of diabetes mellitus in Malawi: population-based national NCD STEPS survey. BMC Endocr Disord. 2014 May 12;14:41. doi: 10.1186/14726823-14-41.PMID: 24884894

7. Msyamboza KP, Kathyola D, Dzowela T. Anthropometric measurements and prevalence of underweight, overweight and obesity in adult Malawians: nationwide population based NCD STEPS survey. Pan Afr Med J. 2013 Jul 24;15:108. doi: 10.11604/ pamj.2013.15.108.2622. eCollection 2013. 